\title{
Metodología de Diseño de Proyectos de Investigación Agraria
}

David Medianero Burga

Resumen: El presente artículo constituye una breve exposición de un trabajo desarrollado en el marco de las actividades del Fondo de Desarrollo de Servicios Estratégicos de INCAGRO. Ofrece una propuesta metodológica para la identificación, formulación y evaluación de proyectos de investigación estratégica agraria. No obstante, su aplicación puede hacerse extensiva a proyectos de investigación científica en otros sectores. La característica esencial de la metodología propuesta es la combinación del método científico - base del proceso investigatorio estratégico- con el método del marco lógico, elemento fundamental del enfoque de proyecto, constituyéndose de este modo en una transacción entre la metodología de investigación y la metodología de diseño de proyectos de inversión. Los temas que se abordan van desde los aspectos netamente conceptuales, como punto de partida que permite establecer en forma clara y sencilla un lenguaje y criterios comunes, hasta la exposición de cada uno de los pasos de la metodología, que comprende la identificación, formulación de los aspectos científicos y programáticos y la evaluación bajo tres modalidades: costo - efectividad, costo - impacto y costo - beneficio.

Palabras Clave: Proyectos, investigación estratégica, marco lógico, diseño experimental, análisis costo efectividad, análisis costo impacto, análisis costo beneficio. 


\section{David Medianero Burga}

\section{Introducción}

El diseño de un proyecto de investigación debe tener en cuenta dos consideraciones fundamentales: una de carácter general, al tratarse de proyectos de inversión; y otra, de carácter particular, al tratarse específicamente de inversiones orientadas a la generación de conocimientos. Las características generales hacen referencia al hecho que, en su calidad de proyectos de inversión, las investigaciones estratégicas constituyen intervenciones temporales, con fechas de inicio y términos predeterminados, que contribuyen a elevar la capacidad productiva y, por ende, los niveles de productividad y competitividad. Tienen, además, un costo determinado por los insumos necesarios para la ejecución de las actividades previstas. De otro lado, las características particulares están relacionadas a la generación de nuevos conocimientos, los cuales contribuyen directa o indirectamente al incremento de la productividad agraria. Estos conocimientos debe anotarse- son obtenidos mediante la utilización del método científico, que esencialmente consiste en la realización de experimentos o estudios observacionales comparativos sustentados en protocolos establecidos en el campo de la investigación científica.

\section{Investigación experimental y marco lógico}

La investigación experimental tiene una característica básica. A diferencia de métodos alternativos de investigación, en un estudio de carácter experimental el investigador manipula la variable o variables independientes antes de medir el efecto sobre la variable dependiente. La manipulación de variables independientes, junto con procedimientos para controlar las variables latentes, forma la base de la eficacia de la investigación experimental. Mientras mayor sea el control del investigador sobre las variables experimentales y variables externas, mayor confianza podrá tener en las relaciones de causalidad en el fenómeno bajo estudio.

Lo anterior, sin embargo, no tiene nada de nuevo. Lo distintivo es talvez puntualizar el hecho que los distintos elementos del proyecto de investigación estratégica pueden ser visualizados a través del instrumento denominado marco lógico. Así, la justificación de la investigación se refleja en el Fin; la hipótesis se refleja en el Propósito; el diseño de la investigación en los Componentes; y el plan de implementación en las Actividades. El marco lógico refleja, grosso modo, el planteamiento de una investigación experimental, en los términos siguientes:

\section{COLUMNA DE OBJETIVOS}

\begin{tabular}{|c|l|}
\hline Fin & $\begin{array}{l}\text { Cadena de eventos emergentes, surgidos como consecuencia } \\
\text { de la obtención del nuevo conocimiento. }\end{array}$ \\
\hline Propósito & $\begin{array}{l}\text { Nuevo conocimiento (variable dependiente), basado en las } \\
\text { hipótesis validadas. }\end{array}$ \\
\hline Componentes & $\begin{array}{l}\text { Tratamientos (variables independientes manipuladas), de } \\
\text { conformidad con un plan experimental. }\end{array}$ \\
\hline Actividades & $\begin{array}{l}\text { Acciones puntuales comprendidas en los tratamientos y } \\
\text { consideradas en el marco del plan experimental. }\end{array}$ \\
\hline
\end{tabular}


Metodología de diseño de proyectos de investigación agraria

Las columnas de Indicadores y Medios de Verificación contienen los datos numéricos y sus fuentes que, en conjunto, forman la línea de base utilizada al inicio de la ejecución y la línea de salida empelada al término de la ejecución.

\section{MARCO LÓGICO DE PROYECTOS DE INVESTIGACIÓN CIENTÍFICA}

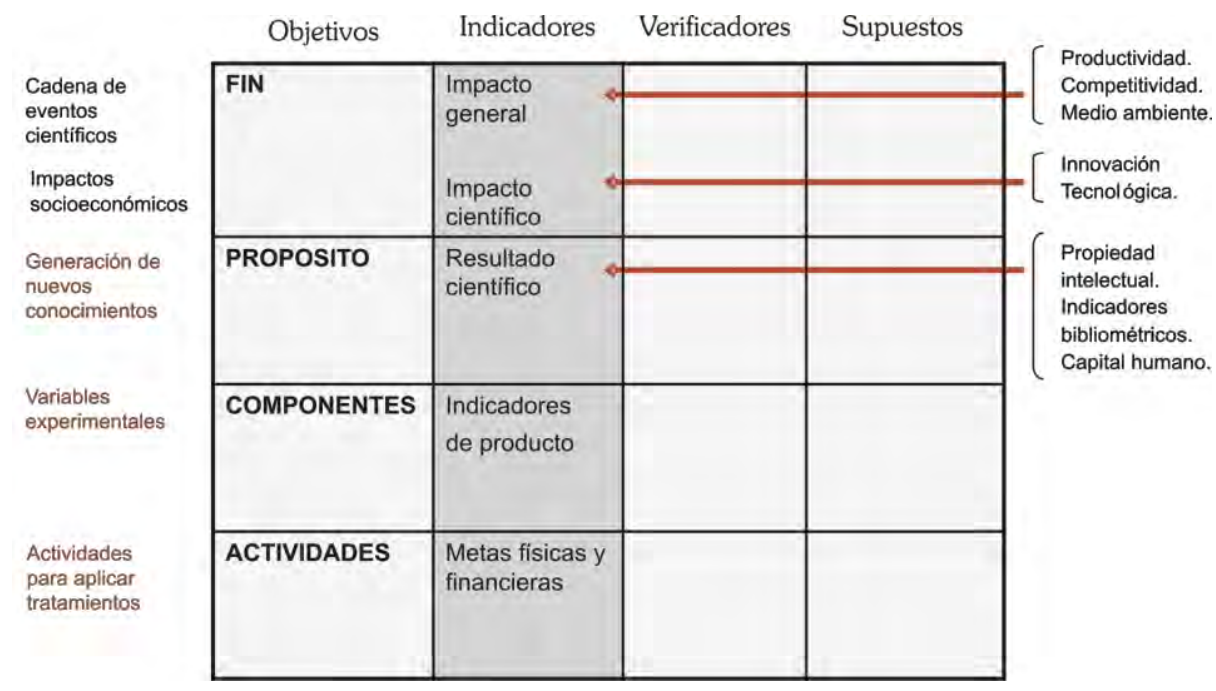

\section{Tipología básica en proyectos de investigación}

Los proyectos de investigación presentan una tipología básica dividida en tres categorías principales: proyectos de investigación básica, proyectos de investigación aplicada y proyectos de desarrollo experimental. Esta es la clasificación clásica, basada en el Manual de Frascati de la Organización de Cooperación y Desarrollo Económico (OCDE), que tiene su equivalencia en los distintos tipos de proyectos de investigación estratégica.

\section{TIPOLOGIA DE LAS INVESTIGACIONES}

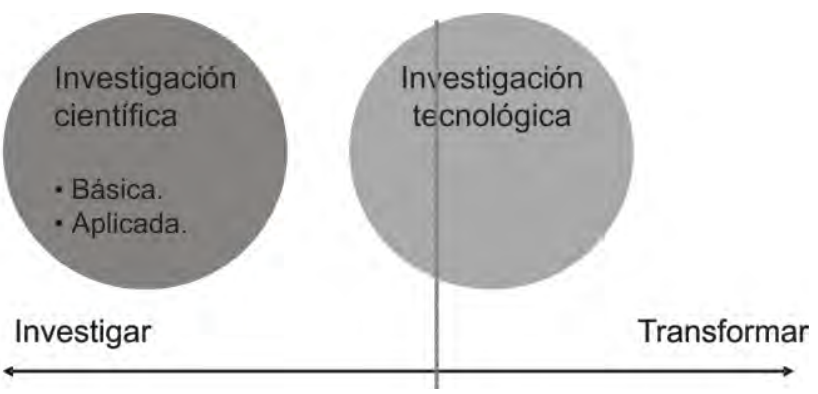




\section{David Medianero Burga}

La característica esencial en estos proyectos es que contribuyen a la generación de nuevos conocimientos. Sin embargo, las diferencias entre unos y otros están relacionadas al tipo de conocimiento que generan. Así, la investigación básica está vinculada al conocimiento científico puro, razón por la cual es conocida también con el nombre de investigación pura o fundamental. La investigación aplicada, por su parte, está vinculada al desarrollo de inventos, sustentados en conocimientos científicos. Finalmente, el desarrollo experimental busca transformar un invento en una innovación de productos o procesos que contribuyen al desarrollo socioeconómico. Esta última tipología también adopta el nombre de investigación tecnológica y cuando toma la forma de proyecto se denomina proyecto tecnológico.

Los proyectos de investigación estratégica pueden referirse a acciones temporales de investigación básica aplicada o tecnológica, con énfasis en estos últimos, en la medida en que presentan un mayor potencial de contribución al incremento de la productividad y competitividad.

En todos los casos, sin embargo, se trata de conocimiento sobre aspectos mesurables de la realidad objetiva, obtenidos mediante la aplicación de métodos observacionales o experimentales en los cuales se ha desarrollado un protocolo de investigación sustentado en el método científico.

Desde la perspectiva del enfoque del marco lógico, por otra parte, los proyectos de investigación estratégica se pueden clasificar en tres tipos básicos: $\mathrm{PIE}_{1}, \mathrm{PIE}_{2}$ y $\mathrm{PIE}_{3}$.

Proyectos PIE1. Son proyectos de investigación estratégica cuyo marco lógico se agota en los resultados de la investigación misma, sin impactos socioeconómicos observables y mensurables.

Proyectos PIE2. Son proyectos de investigación estratégica cuyo marco lógico incorpora impactos socioeconómicos observables y en ciertos modos mensurables.

Proyectos PIE3. Son proyectos de investigación estratégica cuyo marco lógico incorpora a productores beneficiarios dentro de los efectos, además de contar con impactos socioeconómicos observables. 


\section{TIPOLOGIA DE SUBPROYECTOS DE INVESTIGACION ESTRATEGICA}
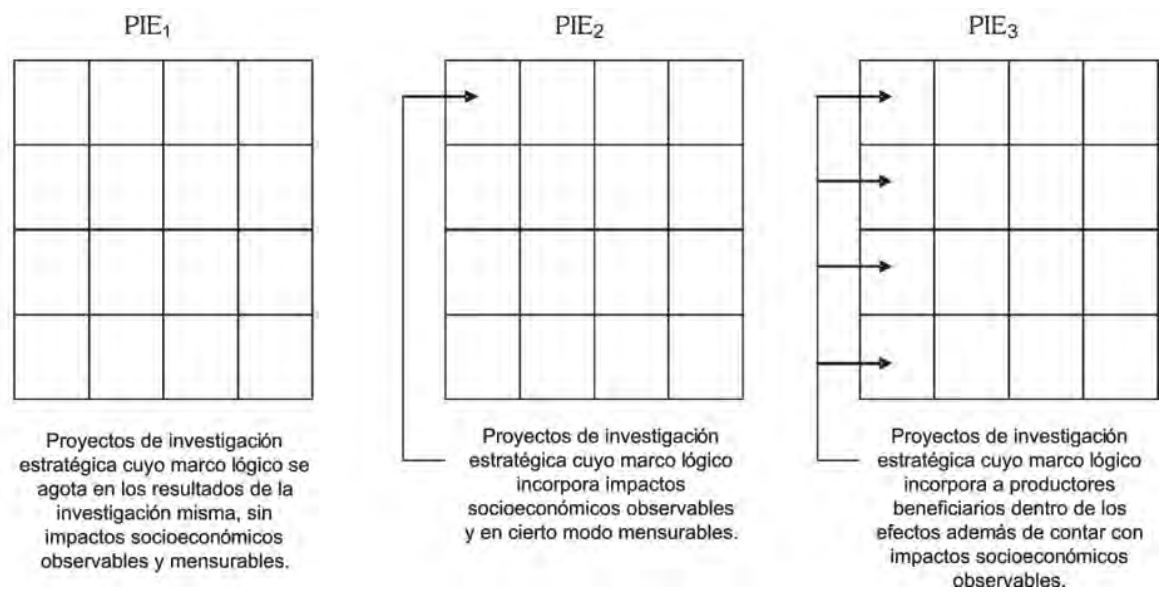

\section{Tipología básica en métodos de investigación}

Así como existen distintos tipos de investigación, también existen distintos métodos de investigación científica, contándose entre los más importantes el método experimental, el método observacional y el método ex post facto.

Método experimental. Consiste en someter deliberadamente un grupo de individuos a algún tratamiento, con el objetivo de observar sus respuestas. En este tipo de estudios suele ser posible asignar relaciones causales entre las respuestas y los tratamientos.

Método observacional. Consiste en medir las variables de interés intentando no influir sobre las respuestas. Cuando muestreamos, el objetivo es obtener información procurando alterar lo mínimo a la población. Los estudios observacionales se limitan a asociar las relaciones entre las respuestas y las condiciones del tratamiento. Los estudios observacionales que tratan de investigar el efecto de una variable sobre otra a menudo fallan debido a que la variable explicativa se confunde con variables latentes.

Estudio ex post facto. La investigación se realiza después de ocurrido el acontecimiento. La confiabilidad de los conocimientos obtenidos por esta vía, sin embargo, es de un grado menor a la de los otros métodos.

Los subproyectos de investigación estratégica agraria suelen ser efectuados mediante la utilización de los dos primeros métodos señalados, debido a su mayor grado de validez y confiabilidad. 


\section{Tipología general de subproyectos de investigación estratégica}

Al combinar la tipología de proyectos de investigación científica con la tipología correspondiente a los métodos de investigación, se obtienen una tipología ampliada que, para el caso de los proyectos de investigación estratégica, está compuesta por seis tipos de proyectos.

* Proyectos de investigación básica con método experimental. Son proyectos que generan nuevos conocimientos estrictamente de carácter científico, que para su obtención requieren de la aplicación de diseños experimentales.

* Proyectos de investigación básica con método observacional. Son aquellos que generan igualmente conocimientos científicos pero que por razones técnicas, éticas, o económicas no pueden ser obtenidos mediante la realización de experimentos, en cuyo caso se recurre a métodos observacionales.

* Proyectos de investigación aplicada con método experimental. Son proyectos en los cuales los conocimientos obtenidos tendrían un impacto socioeconómico directo y serian obtenidos mediante la realización de experimentos.

* Proyectos de investigación aplicada con método observacional. Son proyectos que igualmente generarían conocimientos con impactos socioeconómico directo, pero sin necesidad de realizar experimentos, sino mediante observaciones comparativas de situaciones naturales.

* Proyectos de innovación tecnológica con método experimental. Son proyectos que generan innovaciones de aplicación productiva inmediata, pero que para su validación requieren de la aplicación de experimentos.

* Proyectos de investigación tecnológica con método observacional. Son aquellos proyectos que igualmente generan innovaciones de aplicación productiva inmediata, incluyendo el beneficio de alguna determinada población beneficiaria, y cuya validación ha sido hecha mediante observaciones comparativas de situaciones naturales.

TIPOLOGÍA AMPLIADA DE SUBPROYECTOS DE INVESTIGACIÓN ESTRATÉGICA

\begin{tabular}{|l|c|c|c|}
\hline \multirow{2}{*}{$\begin{array}{l}\text { Métodos de } \\
\text { investigación }\end{array}$} & \multicolumn{3}{|c|}{ Tipos de investigación } \\
\cline { 2 - 4 } & $\mathrm{PIE}_{1}$ & $\mathrm{PIE}_{2}$ & $\mathrm{PIE}_{3}$ \\
\hline \hline $\begin{array}{l}\text { Método } \\
\text { Experimental }\end{array}$ & $\checkmark$ & $\checkmark$ & $\checkmark$ \\
\hline \hline $\begin{array}{l}\text { Método } \\
\text { Observacional }\end{array}$ & $\checkmark$ & $\checkmark$ & $\checkmark$ \\
\hline \hline $\begin{array}{l}\text { Método } \\
\text { Ex-post facto }\end{array}$ & $\times$ & $\times$ & $\times$ \\
\hline
\end{tabular}


Metodología de diseño de proyectos de investigación agraria

\section{Metodología propuesta}

El diseño de un proyecto de investigación estratégica puede ser realizado aplicando una metodología de identificación y formulación que, grosso modo, comprende los 10 pasos siguientes.

1. Identificación del problema de investigación. Consiste en determinar el problema científico o tecnológico que será materia de investigación. El instrumento metodológico sugerido es la Matriz de Selección de Problemas, a través de la cual se realizan comparaciones en el ámbito socioeconómico, en base a criterios de pertinencia, viabilidad e incidencia.

2. Justificación de la investigación. Implica la realización de un análisis inicial de los efectos que tendría la investigación. Para ello, se sugiere el uso de tres tipos de indicadores: indicadores de resultado científico, indicadores de impacto científico e indicadores de impacto general. En determinados casos, la justificación de la investigación implicará un análisis de involucrados y, específicamente, de la población beneficiaria.

3. Formulación de hipótesis. Se realiza sobre la base del árbol de problemas y árbol de objetivos. La hipótesis de investigación es una respuesta tentativa al problema de investigación. En la medida en que se encuentra formulada como relación entre dos o mas variables, es posible utilizar la técnica del árbol, ya sea en su versión negativa (árbol de problemas) o positiva (árbol de objetivos).

4. Análisis de alternativas y elaboración del plan de investigación. Luego de formulada la hipótesis, se diseña la estrategia de investigación, que debe considerar por lo menos dos opciones alternativas de solución al problema investigatorio, formulando luego el plan de experimentación. El plan de investigación puede ser complementado con los aspectos no científicos del proyecto, tales como capacitación del personal de investigación, fortalecimiento institucional y difusión de los resultados de la investigación.

5. Plan operativo del proyecto. Se elabora sobre la base del plan de investigación. El plan operativo contiene los componentes científicos (experimentales y/o observacionales) y no científicos, así como las metas físicas y financieras. Desde la perspectiva del investigador, el plan operativo está constituido por las actividades de investigación. Desde la perspectiva del proyectista, el plan operativo está constituido por todas las actividades: experimentales, observacionales y/o organizacionales.

6. Estimación de beneficios del proyecto. Consiste en identificar los beneficios que generará el subproyecto de investigación en términos de productos y/o servicios, así como de adopción de los resultados de una innovación generada a través de dicho proyecto. Vale decir, se analiza de qué manera impacta al usuario o consumidor final, que en este caso, es el agricultor. Este proceso requiere del análisis de variables como el horizonte del proyecto, la cercanía al 


\section{David Medianero Burga}

mercado (en términos de resultado esperado), la etapa dentro del proceso de investigación, entre otros.

7. Estimación de costos del proyecto. Se realiza para efectos de presupuestación. Comprende los costos de inversión, es decir, los costos de ejecución de todas las actividades y los costos para efectos de evaluación, que comprende los costos directos e indirectos incrementales de operación.

8. Análisis de rentabilidad, sensibilidad y riesgo del proyecto. Consiste en la identificación de la metodología pertinente para la evaluación, pudiéndose elegir entre tres opciones: el análisis costo efectividad, el análisis costo impacto y el análisis costo beneficio. La naturaleza particular de la investigación y el tipo de información que estas pueden proporcionar serán determinantes en la elección de la metodología de evaluación. Este paso incluye analizar el efecto que pueden tener diversas variables que podrían afectar de manera importante la ejecución del proyecto (precios, costos, el indicador de efectividad, entre otros). El análisis de riesgo consiste en evaluar las probabilidades de alcanzar la fase de desarrollo del subproyecto y su producto, alcanzar la fase de transferencia del producto desarrollado por el proyecto y; de alcanzar la fase de adopción de la nueva tecnología generada.

9. Análisis de sostenibilidad. Consiste en la evaluación de los elementos del diseño del proyecto que garantizan la inserción de la investigación propuesta en una cadena de eventos científicos y socioeconómicos que auguran un desarrollo posterior de los conocimientos generados.

10. Marco lógico. Sobre la base del plan experimental de la alternativa seleccionada, se puede elaborar un marco lógico desde dos perspectivas que, si bien son esencialmente similares, guardan algunas diferencias notables. De esta forma se puede elaborar un marco lógico desde la perspectiva del investigador, en el cual la hipótesis se ubica en el propósito, y los componentes experimentales y sus actividades correspondientes se ubican en los casilleros de componentes y actividades del marco lógico, respectivamente. Por otro lado, el marco lógico desde la perspectiva del proyectista ubica dentro de los casilleros de componentes y actividades, además de los aspectos meramente experimentales, aquellos aspectos no experimentales tales como: fortalecimiento institucional, capacitación del personal científico y difusión de los resultados de la investigación científica.

\section{Identificación del problema}

La determinación del problema científico y/o tecnológico que el proyecto intentará solucionar es la decisión más importante del proceso de preinversión. Los proyectos de investigación estratégica son, esencialmente, grandes respuestas a determinados problemas de la producción, en este caso agraria. El error más grave que se puede cometer en el diseño de 


\section{Metodología de diseño de proyectos de investigación agraria}

estos proyectos consiste en intentar resolver con precisión el problema equivocado. Existen diversas formas de efectuar el análisis de la situación actual, siendo el más usado el método participativo denominado lluvia de ideas. Este método permite arribar a un registro de situación; esto es, un listado simple de los principales problemas que aquejan a la población objetivo de la entidad ejecutora y, posteriormente, seleccionar el problema central. En este paso se establecen las fronteras de todo el proceso.

Usualmente, los problemas se identifican al realizar el diagnóstico de la institución o de la localidad de que se trate. Acto seguido, el reto consiste en identificar los problemas que, por su gravedad y relevancia, ameriten su atención en el plazo más breve. El diagnóstico de la situación actual es el proceso sistemático de identificación de los grandes problemas existentes en el ámbito de trabajo de la institución y que, por ende, representan oportunidades para el mejoramiento del desempeño. La situación actual, por lo general, es evaluada a través del uso de indicadores de desempeño, los cuales reflejan la contribución de la organización al desarrollo económico y mejoramiento de la calidad de vida de la población.

Los criterios para plantear adecuadamente un problema de investigación son fundamentalmente tres: El problema debe expresar una relación entre dos o más conceptos o variables; debe estar formulado como pregunta, claramente y sin ambigüedad y finalmente debe implicar la posibilidad de realizar una prueba empírica. El principio general es que un problema debe ser lo suficientemente específico para ser abordado mediante un proyecto y lo suficientemente general para admitir diversas alternativas de solución.

Sobre la base de los problemas identificados mediante la lluvia de ideas, se selecciona el problema central que será revertido con la implementación del proyecto. Para ello resulta de utilidad la elaboración de una matriz, denominada Matriz de Selección del Problema Central, en la cual se comparan los problemas en función a criterios relevantes en cuanto a la implementación o no de un proyecto, tales como: pertinencia, viabilidad e incidencia.

Este paso es indispensable por dos razones: por un lado, la selección del problema central permite un procesamiento mucho más eficaz del análisis del proyecto; y por otro, permite realizar un proceso de aproximaciones sucesivas que supone el análisis de situación, ya que éste sólo se puede efectuar en torno a un problema o núcleo de problemas. El resultado de este proceso es la acotación del listado de problemas a un único problema considerado como el de mayor repercusión en el desempeño de la institución

\section{Justificación de la investigación}

La mayoría de las investigaciones se realizan con un propósito definido y dicho propósito debe ser lo suficientemente significativo como para que se justifique su realización. Con la 


\section{David Medianero Burga}

finalidad de evaluar la importancia potencial de una investigación, se pueden utilizar determinados criterios, que se exponen a continuación en forma de preguntas.

* Conveniencia. ¿Para qué sirve la investigación?

* Relevancia social. ¿Cuál es su trascendencia social?, ¿Quiénes se benefician?

* Implicaciones prácticas. ¿Ayudará a resolver algún problema social?

* Valor teórico. ¿La investigación llenará algún vacío del conocimiento? ¿Puede sugerir ideas o recomendaciones para estudios posteriores?

* Utilidad metodológica. ¿La investigación puede ayudar a crear un nuevo instrumento para recolectar a o analizar los datos?

Respecto a estas preguntas, cabe anotar que cuanto mayor número de respuestas se contesten en forma positiva, la investigación tendrá bases más sólidas para justificar su realización. Para ello el instrumento utilizado es la construcción de indicadores de desempeño. Para el análisis de la importancia de una investigación, pueden ser utilizados tres tipos de indicadores: indicadores de resultado científico, indicadores de impacto científico e indicadores de impacto general.

Indicadores de resultado científico. Se refieren a los resultados de la investigación y, por ende, dependerán de la naturaleza del subproyecto correspondiente. Existen pocas posibilidades de generalización, razón por la cual cada proyecto establecerá los indicadores pertinentes, sobre la base de ciertos criterios generales que se especifican en la siguiente sección. Sin embargo, con sus particularidades, los resultados de la investigación puede ser clasificada en tres rubros: indicadores de propiedad intelectual, indicadores bibliométricos e indicadores de capital humano.

Indicadores de impacto científico, se refieren a la cadena de eventos científicotecnológicos impulsados por el subproyecto, entre los que se pueden destacar:

Generación y aplicación de nuevos conocimientos y tecnologías.

* Formación de recursos humanos.

* Desarrollo de infraestructura científico- tecnológica.

* Creación y mejoramiento de líneas de investigación y desarrollo.

* Articulación de grupos de investigación.

* Integración de nuevos científicos y tecnólogos.

* Mejoramiento de laboratorios e instalaciones de investigación y desarrollo

* Desarrollo de capacidades de gestión tecnológica.

* Aumento en el vínculo de universidades o institutos de investigación con el mundo empresarial. 


\section{Metodología de diseño de proyectos de investigación agraria}

$*$

Publicaciones en prensa especializada.

Indicadores de impacto general, se refieren a los efectos socioeconómicos a los que en forma directa o indirecta contribuye o se espera que contribuya un subproyecto de investigación estratégica. Siguiendo al metodología en vigencia en INCAGRO, se propone la utilización de indicadores relacionados a tres variables clave: productividad, competitividad y medio ambiente.

\section{Planteamiento de la hipótesis}

Las hipótesis son explicaciones tentativas del fenómeno investigado que deben ser formuladas como proposiciones. En el ámbito de la investigación científica, las hipótesis son proposiciones tentativas acerca de las relaciones entre dos o más variables, y se apoyan en conocimientos organizados y sistematizados. Las hipótesis no necesariamente son verdaderas, por lo que el investigador al formularlas no estará totalmente seguro que éstas vayan a comprobarse. En la preparación de un proyecto de investigación estratégica la formulación de hipótesis constituye un paso muy importante, el cual se realiza sobre la base del análisis del problema de investigación. Esto equivale a establecer el objetivo del proyecto, toda vez que la hipótesis constituye una explicación tentativa del problema, y la prueba de la hipótesis constituirá la razón de ser de la investigación. Para efectuar este paso, se puede emplear como instrumentos los árboles de problemas y objetivos.

Las hipótesis de investigación como respuestas tentativas al problema inicial, constituyen una afirmación acerca de la existencia de algún tipo de relación -sea del carácter que fuere-entre dos tipos de variables: una variable independiente y otra dependiente. Para poner a prueba esta relación, el investigador trata de aislar dichas variables a fin de poder afirmar con certeza que efectivamente tal relación existe y que se puede descartar con confianza la injerencia de eventos que habrían podido alterar el proceso de investigación. A tal respecto, se pueden identificar diversos tipos de variables: independientes, dependientes, controladas, extrañas y espurias.

* Variable independiente. Es aquella que se ha identificado como la posible causa o evento promotor de los cambios o del surgimiento de la variable dependiente.

* Variable dependiente. Es a su vez el efecto que se desea estudiar y que se supone es el resultado de la manipulación o aparición de la variable independiente.

* Variables controladas. Son las condiciones que se suponen vinculadas a la variable independiente o a la variable dependiente y que son conocidas por el investigador antes de efectuar la experimentación, de tal forma que se procura eliminarlas o mantenerlas constantes durante el experimento, de manera que no afecten el desarrollo. 


\section{David Medianero Burga}

* Variables extrañas. Son eventos que pueden surgir e incidir en el proceso de investigación, alterando de manera significativa los resultados del mismo.

* Variables espurias. Son variables que, en principio se las clasifica usualmente como independientes, y que aparentemente producen cambios en la variable dependiente, pero que luego, en una evaluación posterior, se descubre que no es ella sino otra variable, la que produce los cambios de la variable dependiente.

Luego de haber definido el problema de investigación, se analizan sus causas y efectos. El punto de partida para solucionar un problema es analizarlo correctamente, con la ayuda de un instrumento metodológico denominado "árbol de problemas". El análisis de efectos consiste en representar gráficamente los efectos surgidos a consecuencia del problema. El procedimiento, en forma general, consiste en colocar en un primer nivel los efectos directos o inmediatos del problema. Cada efecto nace del problema, lo que se representa con una flecha desde el problema hacia cada efecto inmediato. A continuación, se pregunta para cada efecto de "primer nivel" si hay alguno o varios efectos superiores importantes que puedan derivarse de él. De ser así, habrán de ser representados en un segundo nivel, derivándolos con flechas de abajo hacia arriba desde el efecto de primer nivel que opera como causa. Si a un efecto concurre como causa otro efecto de primer nivel ya representado, se indica la interdependencia con una flecha. Se continúa sucesivamente con el método para otros niveles, hasta llegar a un nivel que se considere como el superior dentro del ámbito de competencia o de posibilidades de intervención.

A partir del problema central, hacia abajo, se representan las posibles causas del problema central. Es muy importante tratar de determinar el encadenamiento que tienen estas causas. En particular, es muy importante tratar de llegar a las causales primarias e independientes que estén originando el problema. Mientras más niveles se puedan detectar en el árbol de causas, más cerca se estará de las posibles soluciones a implementar para superar la condición restrictiva que se ha detectado. En el siguiente esquema se muestra el árbol de causas ejemplificado con el proyecto ya señalado. Una vez que se han identificado las causas y efectos del problema central, el paso siguiente es integrarlas en un sólo cuadro, este cuadro representa el resumen de la situación del problema analizado. De este modo, se construye un árbol que tiene como tronco al problema central; como ramas, a los efectos del problema y como raíces a las causas del problema. 
Metodología de diseño de proyectos de investigación agraria

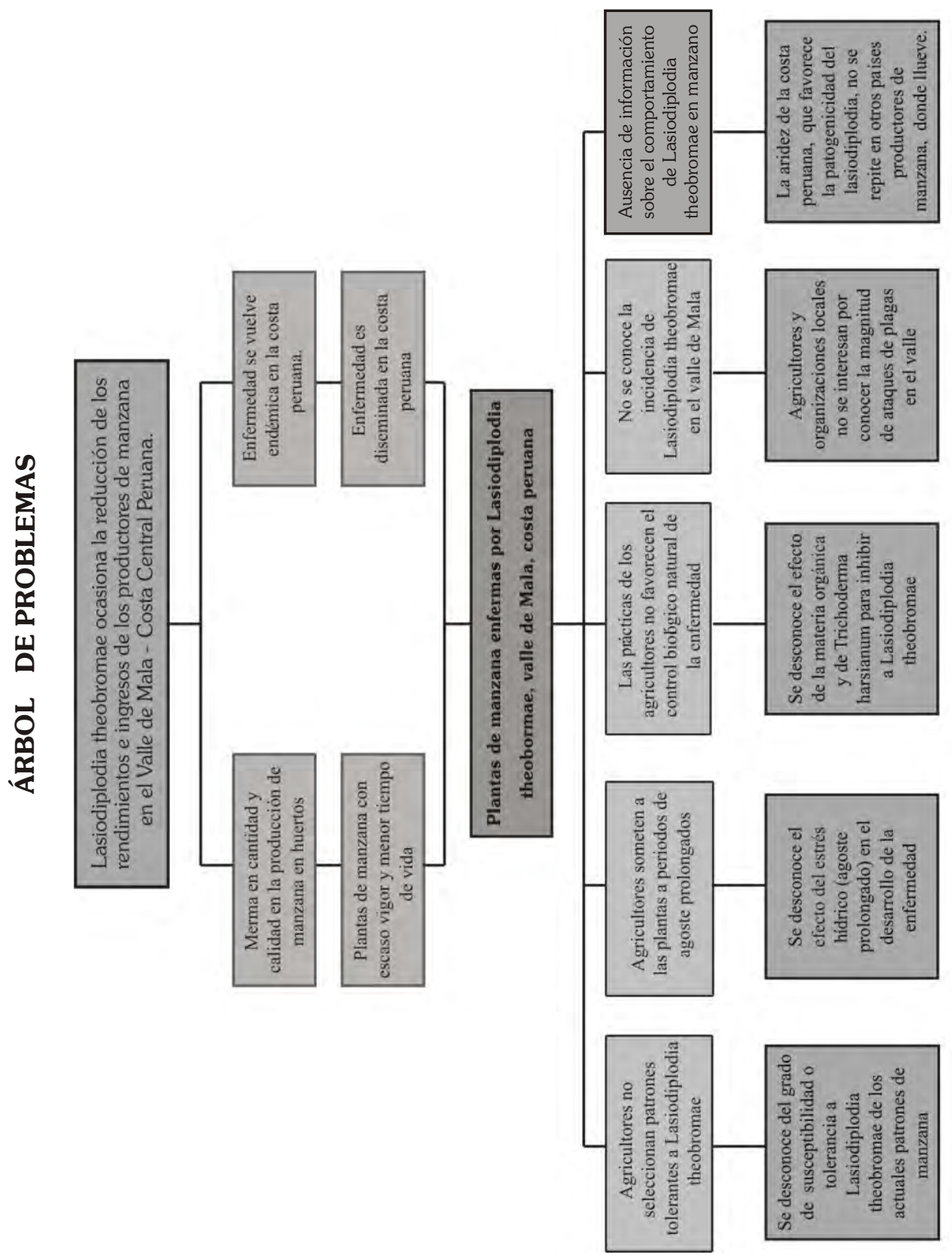




\section{David Medianero Burga}

\section{Análisis de alternativas y formulación del plan de investigación}

Un elemento esencial del enfoque de proyecto es el análisis de las alternativas, del cual se deriva usualmente la elección de aquella alternativa que constituye la solución óptima al problema que el proyecto pretende resolver. En un proyecto de investigación, el análisis de alternativas se refiere a las estrategias o alternativas para la confrontación de las hipótesis, que finalmente se concreta en un diseño o plan experimental, o en el diseño de un estudio observacional. Este diseño, por otra parte, puede expresarse gráficamente a través de un árbol de objetivos.

El diseño experimental constituye el plan o la estrategia para obtener la información que se requiere en una investigación. El investigador debe tratar de seleccionar o desarrollar uno o más diseños de investigación en función de las características propias de su estudio y aplicarlos al contexto particular de su estudio. Esto le permitirá responder de manera práctica y concreta las preguntas de investigación. Debe tenerse en cuenta que un experimento es por definición una situación de control en la cual se manipula de manera intencional una o mas variables independientes (variables causales) para analizar las consecuencias que puede tener dicha manipulación sobre una o mas variables dependientes (efectos) dentro de una situación de control para el investigador

Para la formulación del proyecto de investigación, en lo que se refiere a sus componentes experimentales, se puede utilizar, en calidad de instrumentos metodológicos, el árbol de objetivos y la matriz de selección de alternativas.

Árbol de Objetivos. El árbol de objetivos, en principio, es una versión en positivo del árbol de problemas, donde los medios fundamentales se especifican en el nivel inferior y constituyen las raíces del árbol. En tanto que los fines, más propiamente dicho los objetivos del proyecto, se especifican en la parte superior. El árbol de objetivos es un procedimiento metodológico que permite:

* Describir la situación futura que prevalecerá una vez resueltos los problemas.

* Identificar y clasificar los objetivos por orden de importancia.

* Visualizar en un diagrama las relaciones medios-fines.

De este modo, los estados negativos que muestra el "árbol de problemas" se convierten en estados positivos que hipotéticamente se alcanzarán a la conclusión del proyecto. Cabe destacar aquí que el árbol de objetivos puede realizarse bajo dos perspectivas, que de algún modo resultan equivalentes: el árbol de objetivos bajo la perspectiva del investigador y el árbol de objetivos bajo la perspectiva del proyectista, ambos se derivan del árbol de problemas y como elemento central se basan siempre en la solución del problema principal identificado previamente en el árbol de problemas.

El árbol de objetivos realizado desde la perspectiva del investigador se distingue en que el 


\section{Metodología de diseño de proyectos de investigación agraria}

problema central da origen a una hipótesis básica o central de investigación. Las causas fundamentales, por su parte, pasan a convertirse en los componentes experimentales del proyecto. Así por ejemplo, el árbol de objetivos desde la perspectiva del investigador- del subproyecto "Influencia de los efectos climáticos en los contenidos bioquímicos del yacón" muestra a continuación los elementos que permitirían explicar la fenomenóloga del yacón.

\section{ÁRBOL DE OBJETIVOS - PERSPECTIVA DEL INVESTIGADOR SUBPROYECTO: “INFLUENCIA DE LOS EFECTOS CLIMÁTICOS EN EL YACÓN"}

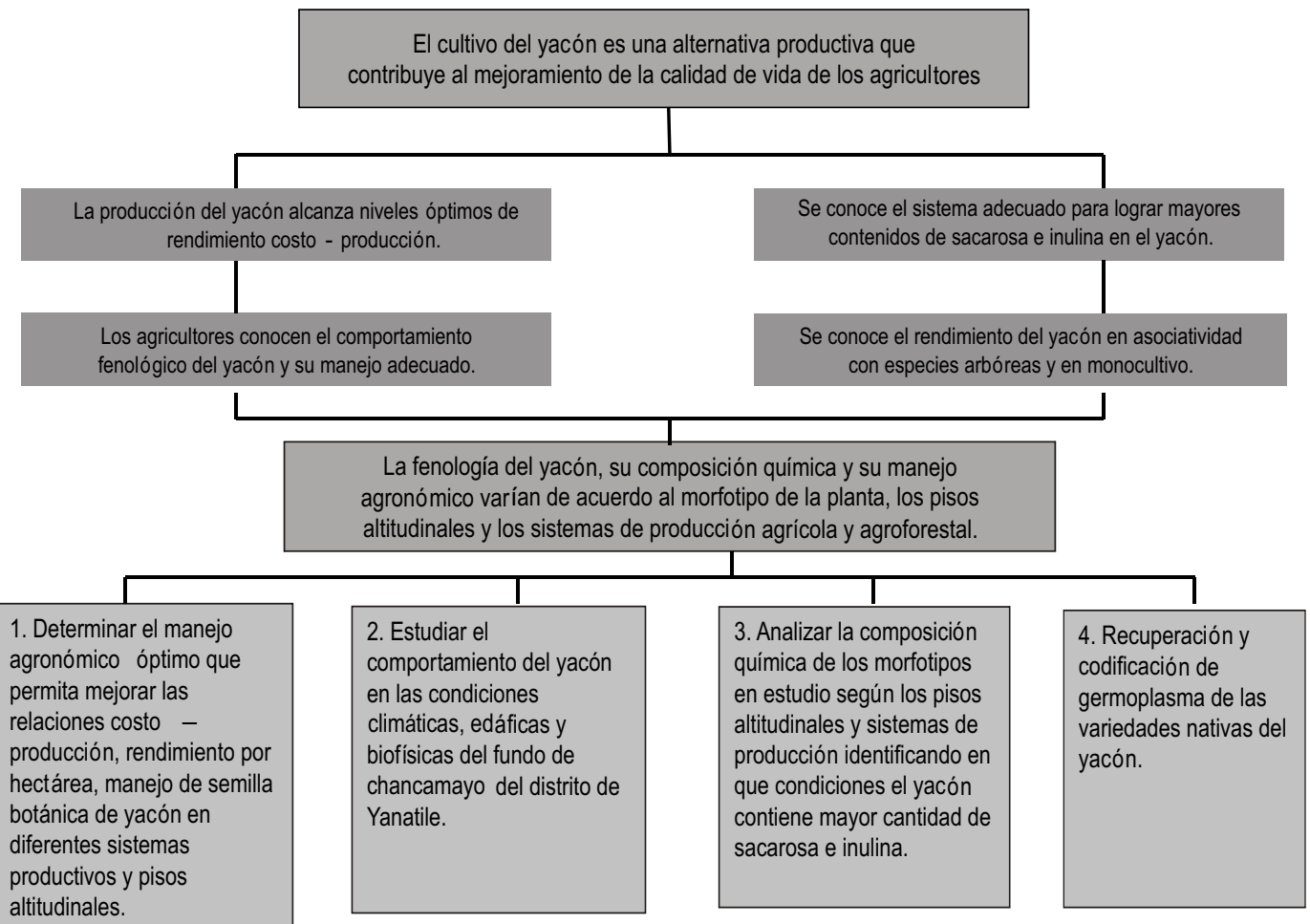

De otro lado, en el árbol de objetivos que se realiza desde la perspectiva del proyectista, el problema central pasa a convertirse en el objetivo central del proyecto, y las causas fundamentales se convierten en los componentes del proyecto social, con la particularidad además de que se considera por lo común un componente usualmente referido al fortalecimiento institucional, que no es de carácter experimental y que hace referencia más bien actividades encaminadas mejorar las capacidades operativas y de gestión de las entidades beneficiarias. El grafico adjunto muestra el árbol de objetivos desde la perspectiva del proyectista- del subproyecto "Influencia de los efectos climáticos en los contenidos bioquímicos del yacón” se muestra a continuación. 


\section{David Medianero Burga}

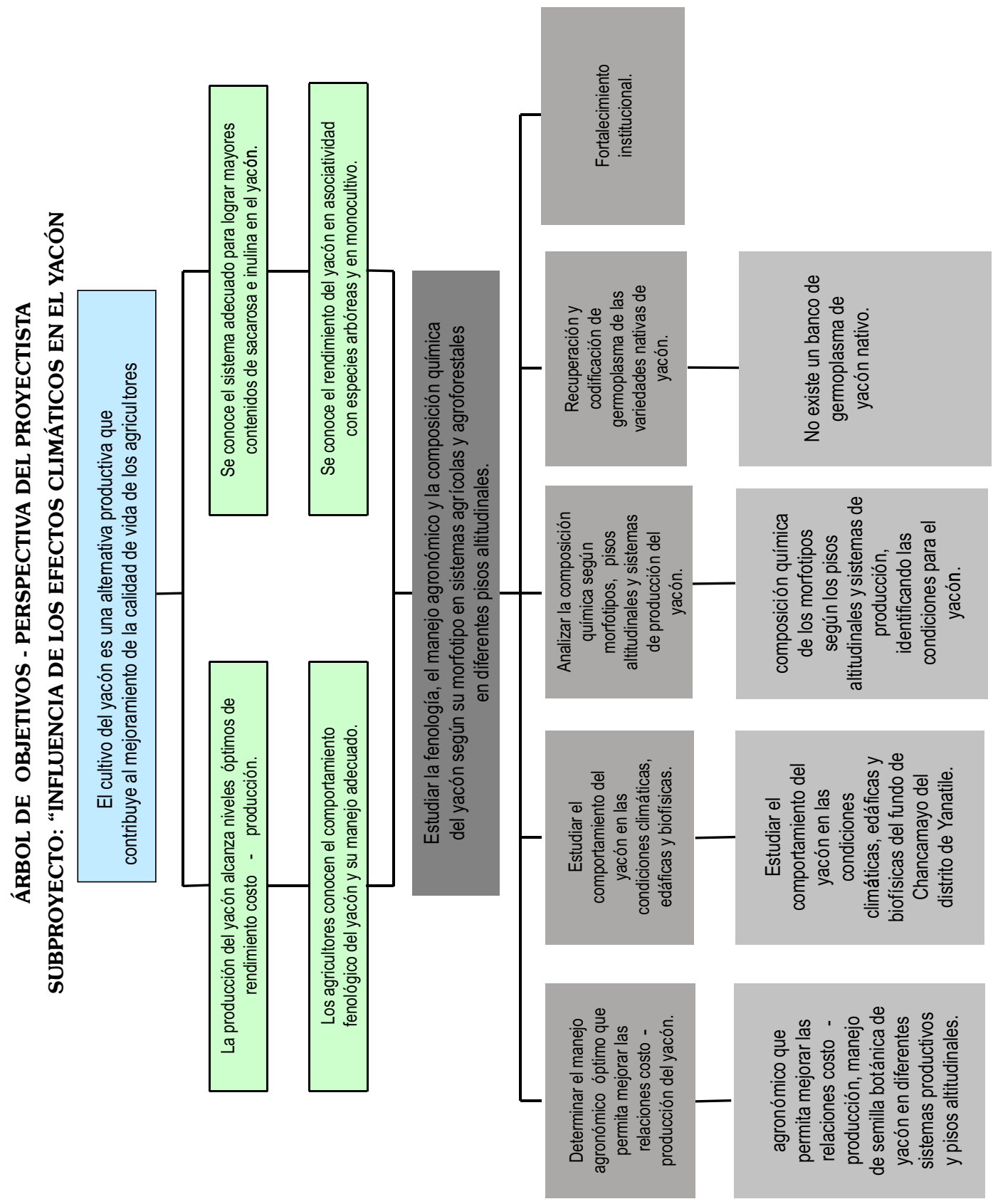




\section{Metodología de diseño de proyectos de investigación agraria}

Matriz de análisis de alternativas. Para la selección de alternativas puede emplearse la matriz de análisis de alternativas, la cual permite realizar tal labor a través de un método de ponderación cuantitativa. Dicha matriz consta básicamente de tres campos: criterios, coeficiente y medios.

* Criterios. En este campo se establece cuáles serán los criterios de priorización de los diferentes medios fundamentales establecidos en el marco lógico.

* Coeficientes. Hace referencia al peso dado a cada uno de los criterios establecidos, a través de una escala que va del 1 al 5 .

* Medios. En este campo se colocan los medios fundamentales establecidos en el árbol de objetivos. Cada uno de los medios recibirá un puntaje, que puede ser mayor o menor según cumpla con cada uno de los criterios establecidos previamente. La puntuación va también de 1 a 5 .

Una vez realizada la puntuación de cada uno de los medios según los criterios, se procede a ponderar dichos puntajes según el mayor o menor peso que posea cada criterio. Los medios que obtengan mayor puntaje son los que satisfacen de mejor manera todos los criterios establecidos. Luego, en el proyecto se podrán seleccionar aquellos medios que, en forma global, hayan obtenido las mayores puntuaciones.

Cabe señalar que en la estructura de una propuesta de investigación científica, existen elementos importantes que tienen repercusiones directas sobre el diseño del plan de investigación. En primer lugar está el problema central, que debe ser adecuadamente identificado, así como sus efectos sobre la población biológica que afecta. Igual importancia tiene la correcta identificación de las causas del problema, que son los factores que en el entendimiento del investigador podrían estar originando el problema en cuestión. Asimismo, otro elemento fundamental es el establecimiento del objetivo general, que por razonamiento lógico, será eliminar o atenuar el problema que afecta a la población. Por último, igualmente importantes son los objetivos específicos, que nacen de las causas probables del problema, las cuales pasan a convertirse en los objetivos de la investigación y de ser el caso, en las hipótesis sobre las cuales hay que tomar decisión con base en la información que proporcione el diseño de la investigación.

La estrategia experimental (o método experimental) en su calidad de estrategia de investigación, se caracteriza por el uso de diseños experimentales para captar información confiable, que permita tomar decisiones sobre las hipótesis planteadas en la investigación, controlando el grado de incertidumbre que involucra esta decisión.

Existen tres diseños experimentales aleatorios básicos: diseño completamente al azar, diseño de bloques completos al azar y el diseño cuadrado latino; los mismos que presentan tres características comunes: i) presentan repeticiones, al tener repeticiones los tratamientos en estos diseños, permiten estimar el error experimental; ii) realizan una asignación aleatoria de 


\section{David Medianero Burga}

tratamientos, que permite darle validez a la estimación del error experimental, y; iii) tienen control local, cuidando de reducir el error experimental, lo cual trata de conseguir al elegir el diseño experimental, al determinar el número de repeticiones que se va ha usar y decidir la forma y tamaño de las unidades experimentales en el caso de experimentos agrícolas. De los tres diseños experimentales básicos mencionados, los más utilizados son el diseño completamente al azar y el diseño de bloques Completos al azar, y cuyos requisitos de uso son los siguientes:

En el diseño completamente al azar la asignación de los tratamientos a las unidades experimentales, se hace completamente al azar sin ninguna restricción, lógicamente este tipo de asignación, solo se podrá hacer si las unidades experimentales son muy similares; las variaciones entre ellas son mínimas. Esta característica hace que este diseño, se utilice muy frecuentemente en ganadería, en donde el investigador puede disponer de un número de animales muy homogéneos en características tales como: sexo, edad, peso, raza. Es decir trata en lo posible que no exista ninguna diferencia entre los animales que van a ser las unidades experimentales del diseño. En el caso de agronomía, estas condiciones de uniformidad, se pueden lograr en ensayos que se van a realizar en laboratorios, invernaderos y viveros.

Por su parte, en el diseño de bloques completos al azar, la asignación de los tratamientos, es también al azar, pero dentro de grupos de unidades experimentales, a los que denominamos bloques. Son "completos" porque dentro de cada grupo existe un número de unidades experimentales igual al número de tratamientos, lo que permite asignar al azar una unidad experimental a cada tratamiento, de manera de tener todos los tratamientos en cada uno de los bloques. Se espera muy poca variabilidad dentro del bloque, pero si existencia de variabilidad entre los bloques y esta forma poder controlar al error experimental. Este y sus variantes es el diseño experimental, de uso más frecuente en la investigación agrícola. Existen otros diseños experimentales como los Latices (simples, cuadrados y rectangulares) que son utilizados por los programas de mejoramiento genético de cultivos y son recomendados cuando el número de tratamientos (entradas) son elevados.

Con relación a la estrategia no experimental, se caracteriza porque se realiza sin manipular de manera intencional las variables independientes (tratamientos). Lo que hace mas bien es observar tal y como se da un fenómeno en su contexto natural para después analizarlo.

\section{Caso 1: Control Ecológico de Lasiodiplodia Theobromae, agente causal del brazo negro en el cultivo del manzano}

A continuación se presenta un caso de evaluación de alternativas de contrastación de hipótesis, realizados sobre la base de la información contenida en los subproyectos presentados al FDSE de INCAGRO. En este proyecto el problema central es la presencia de la Lasiodiplodia Theobromae, hongo parásito con capacidad saprofítica que afecta al cultivo del manzano en la costa peruana. Se estima que este organismo afecta a más del $50 \%$ de los campos en 


\section{Metodología de diseño de proyectos de investigación agraria}

producción, y comprometería entre el $30 \%$ y $40 \%$ de la producción de los mismos. Ello representa entre 3,600 a 4,800 TM de manzana por año. Según el censo del 2005, el valle de Mala, (Lima), siembra 2,500 hectáreas de manzano. Sin embargo esta enfermedad, constituye una amenaza para la producción de dicho cultivo, y por consiguiente para la economía de los productores, problema que se extiende a los agricultores de los valles de Cañete y Huaraz.

Los efectos de esta problemática comprenden el desarrollo de plantas de manzano con escaso vigor y corto periodo de vida, merma en cantidad y calidad en la producción de manzanas, con el consiguiente efecto negativo en la economía de los agricultores, así como la diseminación, al nivel de enfermedad endémica en la costa peruana. Las probables causas de esta situación, que serán analizadas durante la investigación ha realizarse en la zona afectada por el problema, tienen que ver con la nula selección de patrones tolerantes a lasiodiplodia theobromae, la aplicación de periodos de agoste no adecuados, el desconocimiento por parte de los agricultores de medidas de control biológico de la enfermedad, así como también el desconocimiento del efecto del estrés hídrico en el desarrollo de la enfermedad y del efecto de la materia orgánica, como inhibidor de la acción del agente causal de la enfermedad.

\section{El abordaje de las probables causas permitirá definir los objetivos de la propuesta, debiendo generar las hipótesis de investigación.}

El objetivo general es disponer de un conjunto de alternativas que permita reducir la intensidad de la enfermedad denominada "brazo negro" en el manzano y mejorar los rendimientos del cultivo. El objetivo general, es la solución total o parcial del problema central. A partir de esta propuesta y teniendo en cuenta las probables causas, se establecen las estrategias de investigación más apropiadas para cada uno de los objetivos específicos. Estas estrategias son las que constituyen los componentes del plan de investigación

La evaluación técnica, será básicamente el análisis de la adecuación que debe existir entre el objetivo de la investigación y la estrategia de investigación escogida para ello.

Para facilitar la evaluación, se pondrán juntos el objetivo de la investigación y el componente del plan de investigación propuesto con ese fin, analizando la pertinencia de esa decisión. El primer objetivo específico busca identificar el patrón local (membrillo) más tolerante a lasiodiplodia theobromae, para lo cual se plantea la evaluación de un ensayo con 4 tratamientos (dos tratamientos de membrillo con estaca y otros dos de membrillo con estaca en sustrato inoculado), que son comprendidos en el componente 1 del plan experimental. El diseño experimental planteado es el diseño completamente al azar, con cinco repeticiones, donde la variable a estudiar es el grado de la sintomatología presentada en la estaca.

El segundo objetivo específico se orienta a identificar los periodos de agoste que dañan a la planta favoreciendo el desarrollo de la enfermedad. Para ello se plantea la evaluación de dos experimentos, diferenciados por la modalidad de inoculación (inoculación a la rama o al suelo) 


\section{David Medianero Burga}

tal como se detalla en el componente 2 del plan experimental. El segundo componente comprende dos experimentos. En el primero, de diseño completamente al azar con 5 repeticiones, consiste en la inoculación a la rama a través de cuatro tratamientos (1,2 y 4 meses de agoste y un tratamiento sin agoste), donde la variable por estudiar es la longitud de avance de la pudrición en la rama inoculada. El segundo experimento, de diseño completamente al azar con 5 repeticiones, consiste en la inoculación en el suelo a través de cuatro tratamientos (1,2 y 4 meses de agoste y un tratamiento sin agoste), donde las variables por estudiar son la longitud de raíces y el porcentaje del sistema radicular afectado por la pudrición. En ambos experimentos se reaislará el fitopatógeno.

El tercer objetivo específico busca identificar tratamientos orgánicos que inhiban el desarrollo del agente causal de la enfermedad. Para ello, el tercer componente del plan de investigación, plantea la instalación de 2 experimentos en campos comerciales. El primer experimento, de diseño completamente al azar con 4 repeticiones, estudiará el grado de incidencia de la enfermedad en el brote proveniente de la rama podada en forma sanitaria, realizándose para ello cuatro tratamientos (incorporación de guano, incorporación de compost, incorporación de guano más urea y un tratamiento testigo). El segundo experimento, de diseño experimental completamente al azar con 4 repeticiones, busca estudiar el nivel de incidencia de la enfermedad en el sistema radicular que incide en la longitud de las raíces. Para ello se seleccionará una enmienda de trichoderma harzianum en cuatro tratamientos $(0.0 \mathrm{~kg} /$ planta, $0.1 \mathrm{~kg} /$ planta, $0.5 \mathrm{~kg} /$ plantay $1.0 \mathrm{~kg} /$ planta)

Durante la investigación de los efectos producidos en los objetivos 1,2 y 3 se ha utilizado una estrategia de investigación experimental, ya que la naturaleza de los factores en estudio permite

su manipulación por parte del investigador para generar los tratamientos a ser evaluados.

Igualmente las características del material experimental permiten su asignación al azar a los tratamientos, de acuerdo con los requerimientos del diseño experimental escogido, en este caso el "diseño completamente al azar".

El cuarto objetivo específico busca fortalecer la capacidad de las entidades proponentes y colaboradoras para desarrollar un sistema local de diagnóstico y evaluación de problemas productivos. El quinto objetivo específico por último, está dirigido a difundir las alternativas de control para Lasiodiplodia

Theobromae. Para los objetivos 4 y 5 se ha establecido una estrategia, comprendida en el componente 4 del plan de implementación, que contempla

la estructuración y validación del sistema de diagnóstico y evaluación, elaboración de línea base, selección de indicadores prácticos, validación de metodología y talleres de capacitación.

Como se puede apreciar, para estos objetivos específicos se ha diseñado una estrategia no experimental.

La población beneficiaria comprende a los grupos sociales que se beneficiarán de manera directa de los resultados esperados de esta propuesta de investigación estratégica. Incluye en 


\section{Metodología de diseño de proyectos de investigación agraria}

primer lugar a las cerca de 3,000 familias del Valle de Mala que trabajan en el cultivo del manzano, las cuales contarán con un conjunto de alternativas de control del Lasiodiplodia Theobromae que les permitirá mejorar sus rendimientos y con ello sus ingresos. En segundo lugar, las familias dedicadas al cultivo del manzano en costa central que conducen aproximadamente 7,000 Has de este cultivo. Finalmente, están comprendidos también los productores de frutales de la costa en general, que se están viendo afectados por la acción de este hongo polífago.

El análisis de este caso, determina que puede ser categorizado como un proyecto de investigación estratégica cuyo marco lógico incorpora impactos socioeconómicos, observables y en cierto modo mensurable $\left(\mathrm{PIE}_{2}\right)$.

\section{Metodologías de evaluación}

El enfoque de evaluación válido para los proyectos de inversión pública, dentro de los que se incluye a los proyectos de investigación estratégica, es el de evaluación social de proyectos, que se orienta en última instancia a maximizar el bienestar de la sociedad en su conjunto. Para operativizar la evaluación social de proyectos se pueden utilizar distintas metodologías, siendo las mas importantes las cuatro siguientes: análisis costo efectividad, análisis costo impacto, análisis costo beneficio y análisis multicriterio. La metodología de análisis costo efectividad es la que menos información requiere, pero también es la que menos capacidad tiene para evaluar los impactos o beneficios de los proyectos, mientras que la metodología del análisis multicriterio es la mejor en términos de la evaluación de beneficios, pero el inconveniente es que requiere abundante información, lo cual puede elevar sustancialmente los costos de la evaluación. A continuación se realiza un análisis detallado de cada metodología.

El análisis costo efectividad es una técnica de evaluación de proyectos que se utiliza cuando no es posible cuantificar monetariamente los beneficios del proyecto bajo análisis. Se trata de comparar los costos de un proyecto con los beneficios resultantes, pese a que no están expresados en la misma unidad de medida; los costos son usualmente traducidos a unidades monetarias, mientras que los beneficios se traducen en indicadores de objetivos relevantes. La intención es calcular el costo por unidad promedio de beneficio de cada proyecto alternativo, donde la unidad de beneficio se refiere al número de personas, atenciones y casos resueltos, entre otros. Estas unidades de beneficio también se conocen como indicadores de efectividad, y ciertamente dependerán de la naturaleza específica de cada proyecto. El objetivo es seleccionar la alternativa de inversión más eficiente (es decir, menos costosa) entre distintos proyectos alternativos, bajo el supuesto básico de que cualquiera de los proyectos alternativos va a permitir alcanzar los beneficios propuestos. Operativamente, se requiere definir un indicador de efectividad y luego estimar el ratio costo efectividad, para determinar la mejor alternativa.

Los pasos a seguir implican primero calcular los costos privados de cada una de las 


\section{David Medianero Burga}

alternativas de solución al problema. Luego se estiman los costos sociales (de inversión y de operación y mantenimiento), considerando las distribuciones en bienes nacionales, bienes transables, mano de obra, transferencias, entre otros). A continuación se estima el valor actual de los costos sociales netos utilizando la tasa social de descuento, para cada alternativa. Seguidamente, se define un indicador de efectividad, que permita medir los logros del proyecto. Se calcula el ratio costo efectividad para cada una de las alternativas de proyecto y finalmente se selecciona el proyecto a ejecutar, bajo el supuesto de eficiencia, es decir, escogiendo la alternativa menos costosa.

Uno de los aspectos más importantes para la aplicación de esta metodología es la definición de los indicadores, ya que se requiere que reflejen los objetivos y metas del proyecto. Se puede utilizar indicadores de procesos o de impacto, dependiendo del tipo de información disponible y de los objetivos de la evaluación, ya sea tomar decisiones de corto plazo (proceso) o de largo plazo (impacto). El uso de un indicador a nivel de proceso o impacto, dependerá de las características específicas de cada subproyecto bajo análisis. Debe mencionarse que los proyectos de investigación tienen una serie de indicadores científicos que deben ser tomados en cuenta al momento de analizar lo que se espera del proyecto. Estos indicadores se diseñan expresamente para evaluar la calidad de la investigación (Cadena, Waissbluth, Solleiro, 1989). Definido el indicador de efectividad (IE), se define el ratio costo efectividad, de la siguiente forma:

$$
C E=\frac{V A C S N}{I E}
$$

Donde:

CE : $\quad$ Ratio costo efectividad

VACSN : Valor actual de costos sociales netos.

IE : Indicador de efectividad

Es decir, el ratio CE indica el costo promedio por unidad de beneficio (definido a través del indicador). Como se está buscando la alternativa de mínimo costo, se escogerá la alternativaj siempre y cuando se cumpla que:

$$
C E_{j}<C E_{k} \quad \forall k \neq j \quad \text { donde } \mathrm{k}=1 \ldots . \mathrm{n} \text { (número de alternativas) }
$$

Debe señalarse que para realizar una adecuada selección, el indicador de efectividad debe ser el mismo entre las alternativas, de tal manera que se garantice la posibilidad de comparación. Una de las limitaciones de esta metodología es que no es útil para definir la conveniencia de hacer o no hacer un proyecto específico, sino que sólo sirve para comparar entre alternativas de proyectos. En caso no existan alternativas de proyectos, entonces, deberá compararse el ratio de costo efectividad con algún parámetro regional o nacional, que permita definir si se está realizando un buen uso de los recursos. De otro lado, esta metodología tampoco 


\section{Metodología de diseño de proyectos de investigación agraria}

es útil para comparar proyectos o programas que tienen objetivos diferentes, a menos que se les homogenice a través de un indicador común.

El análisis costo impacto, de otro lado, es una metodología muy similar al análisis costo efectividad, en la medida en que se requiere definir también un indicador, pero en este caso se refiere fundamentalmente a indicadores de impacto o de largo plazo. Formalmente, se puede construir un indicador de costo impacto de la siguiente manera:

$$
C I=\frac{V A C S N}{\text { Objetivo }^{*} 100}
$$

Donde:

$\mathrm{CI}$

VACSN

\section{Ratio Costo Impacto}

Valor Actual de los Costos Sociales Netos.

El indicador de costo impacto se lee como el "costo de un $1 \%$ de efectividad". Por ejemplo, si se está tratando de un proyecto cuyo VACSN es de S/. 100000 y que reducirá el porcentaje de producción afectada por la mosca de la fruta de $25 \%$ a $20 \%$, entonces, el CI será:

$$
C I=\frac{100000}{0.05 * 100}=20000
$$

Es decir, reducir en un $1 \%$ la producción que es afectada por la mosca de la fruta cuesta S/. 20000 . Al igual que en el caso del análisis costo eficiencia, se elegirá la alternativa que tenga un menor indicador de costo impacto. También se puede construir un indicador considerando el impacto como numerador y el costo como denominador. En este caso, la definición de la mejor alternativa se hará considerando aquella que tiene el mayor indicador, ya que será la que logre un mayor impacto por unidad monetaria gastada. Al igual que en el caso de la metodología costo eficiencia, este indicador no sirve para determinar la conveniencia de hacer o no un proyecto, sino sólo es útil para definir la mejor alternativa, dentro de un conjunto de alternativas posibles.

En tercer lugar, el análisis costo beneficio es una metodología que compara los beneficios y los costos de una alternativa de proyecto y si los primeros exceden a los segundos, entonces, el proyecto puede ser considerado para su ejecución. $\mathrm{Si}$, en cambio, los beneficios son menores que los costos, el proyecto debe ser rechazado. Cuando existen varias alternativas de proyectos, el análisis costo beneficio permite establecer las diferencias entre los beneficios y costos de cada uno, determinando un rango u orden entre ellos. En el análisis costo beneficio se utilizan los indicadores clásicos de decisión para la ejecución de un proyecto, es decir el VAN y la TIR. Sin embargo, en el contexto de la evaluación social de proyectos de inversión pública que se trabaja en el SNIP, se utiliza el concepto de valor actual neto social, que se define como:

$$
V A N S=\sum_{t=1}^{n} \frac{F B S T_{t}-F C S T_{t}}{(1+T S D)^{t}}
$$




\section{David Medianero Burga}

Donde:

VANS : Valor Actual Neto Social.

FBST : Flujo de Beneficios Sociales Totales en el período t.

FCST : Flujo de Costos Sociales Totales en el período $t$.

TSD : Tasa Social de Descuento.

Ahora, los beneficios sociales netos pueden ser de tres tipos: beneficios directos, beneficios indirectos y beneficios intangibles. Los beneficios directos se refieren a los beneficios y costos directamente generados por la operación del proyecto. Ejemplo: ahorro del tiempo en un proyecto de transporte. Los beneficios indirectos hacen referencia a los beneficios y costos que afectan indirectamente al proyecto; pero pueden ser tan significativos que superen a los directos. En general, se trata del componente ambiental. Los beneficios intangibles son aquellos que resultan muy difíciles o costosos de medir y valorar, debido a que el concepto mismo no se puede valorar o porque no es posible cuantificar monetariamente el beneficio o costo implícito. En este grupo se incluyen factores políticos, geopolíticos, distribución geográfica, entre otros. En la actualidad, la gran mayoría de proyectos no incluye estos conceptos dentro de su evaluación, debido a que no existe una metodología cualitativa que los incluya.

Se supone que en el FBST se deberá incluir los beneficios directos e indirectos que se pueden valorizar y que se produzcan por la ejecución del proyecto. Sobre la base del valor estimado del VANS, los criterios de decisión son:

$\mathrm{Si}: \quad$ VANS $>0$, se puede ejecutar el proyecto.

Si: $\quad$ VANS $<0$, se debe rechazar el proyecto.

$\mathrm{Si}: \quad \mathrm{VANS}=0$, se es indiferente entre ejecutar o no el proyecto.

Debe recordarse que este criterio de decisión se realiza sobre la base de la estimación social de los beneficios y costos, que es el que se realiza desde el punto de vista de la sociedad; un análisis similar, pero a precios de mercado, puede ser realizado por los agentes privados, para definir si invierten o no en el proyecto. Los pasos para aplicar el análisis costo beneficio a los proyectos en el marco del SNIP son: primero, identificar y cuantificar los beneficios sociales o impactos que se pueden atribuir a la ejecución de la alternativa de proyecto (beneficios); segundo, valorizar los insumos necesarios para la ejecución del proyecto, valorizados a los precios sociales (costos sociales); tercero, estimar el valor actual neto social como la diferencia entre los beneficios (directos e indirectos) y costos, considerando la tasa social de descuento correspondiente; $y$, cuarto, seleccionar el mejor proyecto alternativo, considerando los criterios antes mencionados.

El mayor problema que se presenta dentro de la aplicación del ACB es la estimación de los beneficios sociales. En muchos casos, no es posible encontrar precios sociales para la estimación 


\section{Metodología de diseño de proyectos de investigación agraria}

ya que no existen mercados completos o existen fallas de mercado. Al respecto, existe una serie de metodologías de valoración que pueden ser útiles en este contexto.

Mandar en la estimación de precios hedónicos, costos de viaje y daño evitado.

Aunque estas metodologías tienen un mayor desarrollo en el campo de la economía ambiental y de recursos naturales, se reconoce su utilidad en la evaluación social de proyectos

La metodología de evaluación basada en el análisis multicriterio, finalmente, supera gran parte de la crítica a las metodologías analizadas previamente, especialmente a la que refiere que los criterios de decisión utilizados se basan en un solo indicador (de efectividad, de impacto o económico), aún cuando los proyectos afectan un conjunto de variables y que, por lo tanto, el tratar de evaluarlas a través de un solo indicador o criterio, puede sesgar los resultados de la evaluación. En particular, en muchos casos los proyectos, además de buscar maximizar los beneficios económicos (lo que se logra a través de la maximización del VAN Social) también se quiere minimizar el impacto ambiental negativo, maximizar la descentralización territorial, redistribuir progresivamente el ingreso, entre otros. Aunque en el ACB es posible incluir una valorización monetaria de estos objetivos, algunos autores consideran que ese proceso es muy subjetivo y por tanto no lo consideran apropiado y prefieren proponer un mecanismo que permita lograr todos estos objetivos a la vez, que es la evaluación multicriterio.

Los métodos de evaluación y decisión multicriterio comprenden la selección, entre un conjunto de alternativas factibles, de aquella opción que permita la optimización con varias funciones objetivo simultáneas y un único agente decisor, y procedimientos de evaluación racionales y consistentes. El proceso de análisis multicriterio requiere de: i) Un conjunto de alternativas estable, perfectamente identificadas, aunque sin necesariamente conocer todo el análisis cuantitativo y cualitativo; ii) Un conjunto de criterios de evaluación (atributos, objetivos) que permitan evaluar cada alternativa, considerando ponderaciones que reflejen la importancia relativa de cada criterio. El conjunto de criterios debe cumplir con las propiedades de exhaustividad, coherencia, no-redundancia, operacionalidad, mensurabilidad y economicidad; iii) Una matriz de decisión o de impactos que resuma la evaluación de cada alternativa considerando cada criterio. La evaluación puede ser cuantitativa o cualitativa y las medidas pueden expresarse en las escalas cardinal, ordinal o nominal; iv) una metodología o modelo de agregación de preferencias, para definir la alternativa mejor evaluada y; v) Un proceso de toma de decisiones. Se evalúan las características del proyecto de acuerdo con ciertos criterios y valoraciones y se define un indicador que mediante sumas o multiplicaciones aglutina todas las características del proyecto, para posteriormente construir un ranking de proyectos (a esto se conoce como scoring models).

Aunque esta puede ser la metodología que permita aglutinar una serie de criterios 


\section{David Medianero Burga}

importantes al momento de evaluar un proyecto, la gran limitación que tiene es la que se refiere a proponer un mecanismo de agregación (es decir, las ponderaciones) que sea aceptado por todos los agentes y que refleje las condiciones en la cuales la sociedad valora cada uno de las características del proyecto. Del análisis realizado acerca de la tipología de los proyectos de investigación estratégica y de las metodologías de evaluación, puede establecerse una relación entre ellas, que grosso modo, seria la siguiente:

\begin{tabular}{|c|c|c|c|}
\hline & PIE 1 & PIE 2 & PIE 3 \\
\hline Análisis costo efectividad & Aplicable & No aplicable & No aplicable \\
\hline Análisis costo impacto & No aplicable & Aplicable & No aplicable \\
\hline Análisis costo beneficio & No aplicable & No aplicable & Aplicable \\
\hline
\end{tabular}

La aplicación de las metodologías, dependerá de la información disponible y/o de los supuestos que se puedan realizar. 
Metodología de diseño de proyectos de investigación agraria

\section{BIBLIOGRAFÍA Y NOTAS}

* Manual de Frascati (OCDE).

* Según Kerlinger y Lee (2002).

* Proyecto presentado por el Instituto Huayuná al Fondo de Desarrollo de Servicios Estratégicos de INCAGRO.

* Fondo de Desarrollo de Servicios Estratégicos.

Se pueden tomar como ejemplos, los presentados como indicadores de impacto general o científico del Cuadro $\mathrm{N}^{\circ} 6$.

* Valor Actual Neto.

* Tasa Interna de Retorno.

* Sistema Nacional de Inversión Pública.

Para mayor detalle sobre los métodos de estimación, revisar Azqueta (1994).

* Sección basada en Martínez y Escudey (1998). 